\title{
TSPO binding may also represent 'resting' microglia
}

\author{
Lars Farde $^{1}[0$
}

Received: 18 November 2015/Accepted: 19 November 2015/Published online: 30 November 2015

(C) Italian Association of Nuclear Medicine and Molecular Imaging 2015

Inflammation has since long been identified as part of the pathophysiology of the majority of human diseases, and over the last decades it has become clear that it also has a role in several major CNS disorders. Despite lack of a clear definition, the concept "neuroinflammation" has become widely used in the literature as well as in grant applications. In molecular brain imaging, neuroiflammation has become almost synonymous with an increased signal for radioligand binding to the TSPO protein expressed by microglia cells.

Initially, TSPO binding was thought to represent a signal for 'activated' microglia and this view is still prevailing in the literature. Taken to its extreme, it implies that TSPO is a functional marker expressed only by those microglia cells involved in pro-inflammatory or anti-inflammatory activities, but not expressed on "resting" microglia. There are good reasons to question this assumption.

The concept "activated microglia" was based on histological and biochemical observations showing that inflammatory challenges lead to structural changes of microglia cells with release of cytokines and chemokines. In contrast, "resting microglia" were thought to be dormant cells. However, several lines of evidences support the view that microglia provide a TSPO signal also in the healthy human brain. In an important study using two-photon imaging, Nimmerjahn et al. [1] could demonstrate that microglia cells are very dynamic also in the resting state. They serve as housekeepers continually overviewing the adjacent environment using motile processes and protrusions.

Lars Farde

lars.farde@ki.se

1 Department of Clinical Neuroscience, Centre for Psychiatry Research, Karolinska Institutet, Stockholm, Sweden
The functional role of the $16 \mathrm{kD}$ TSPO protein remains to be understood in more detail. It is known that this protein is part of a larger protein complex mounted on the outer membrane of mitochondria, where it has a role in cholesterol transport and subsequent steroid synthesis. A large step forward was the crystallization of the TSPO protein reported in Science, 2015, by two independent centers. X-ray analysis of the crystals indicated that the protein contained five transmembrane helices. Li et al. identified a binding pocket for cholesterol, whereas Guo et al. reported a distinct site for porphyrines. These observations support the notion that the TSPO protein also has a functional role in intracellular pathways under normal physiological conditions.

Therefore, the extreme view that only activated microglia express TSPO probably is not correct. Resting microglia are active housekeepers in need of intact basic mitochondrial functions. Moreover, whereas the transition from a resting to an activated state is rapid, protein synthesis and formation of mitochondria require several hours or days. Therefore, it is likely that the TSPO signal obtained by PET represents both states.

In analyses of hitherto developed radioligands for TSPO, a general observation has been that a specific binding compartment can be identified using two-tissue compartment models. The nature of this compartment has been clarified in a pharmacological PET study by blocking TSPO binding with the unlabeled TSPO ligand XBD173 [2]. It is important to note that only about $60 \%$ of total [11C]PBR28 binding could be blocked by XBD173. Taken together, it cannot be excluded that the TSPO signal simply represents cell count rather than "activation". If upregulated, the signal may thus reflect migration of microglia to an inflammatory site.

The early days of TSPO imaging faced two challenges. Initially, it was discussed whether PET radioligands 
actually could reach intracellular targets. Importantly, cell membranes share a common structural organization: bilayers of phospholipids, and this applies also to the blood-brain-barrier (BBB). Early data obtained with both TSPO ligands and other radioligands for intracellular transporters have been of more general importance since they strongly support the view that radioligands that rapidly pass the $\mathrm{BBB}$ will also be able to rapidly reach intracellular targets in the brain.

Another and more important challenge is the lack of a reference brain region devoid of the targeted TSPO protein. In contrast to cells expressing other proteins, such as several of the G-protein coupled receptors, microglia are everywhere. This means that there is no reference region in the brain that can be used as an estimate for free and nonspecific binding. The traditional approach is to overcome this problem using a metabolite corrected arterial input function. However, the generation of such an input function is itself associated with noise, and data published so far indicate that test-retest reproducibility of TSPO imaging is not as good as for targets where a suitable reference region is available.

A majority of the radioligands that bind to TSPO are sensitive to a well-described single point mutation. To overcome this problem, several other proteins are now considered as potential targets for molecular imaging of microglia. The P2X7 receptor belongs to the family of purinoceptors and is a ligand-gated cation channel that opens in response to ATP. This ion channel is specifically involved in the pro-inflammatory activity of microglia. The P2X7 receptor is of interest for the pharmaceutical industry, since it has been suggested that antagonists may reduce inflammation and, thereby, progression of neuronal damage. Therefore, P2X7R is a potential molecular target for diagnostic purposes as well as for novel drug treatments. Interestingly, within the EU FP7 Concerted action INMiND (Imaging of neuroinflammation in neurodegeneration), promising candidate radioligands for the $\mathrm{P} 2 \mathrm{X} 7$ receptor have been developed recently. It is likely that the successful development of a P2X7 ligand will overcome the problem of sensitivity to polymorphism, but it will not circumvent the problem of lack of a reference brain region suitable for simplified quantification of microglia.

Inflammatory processes in the brain are complex and reach beyond microglial function, thereby offering potential imaging targets not necessarily related to microglia. Pro-inflammatory chemokines (chemotactic cytokines) are responsible for promoting the chemotaxis of leukocytes, i.e. neutrophiles, monocytes and lymphocytes, to inflammatory sites. In the CNS, there are two G-protein-coupled chemokine receptors, the $\mathrm{CC} 2$ receptor (CCR2) that binds the CCL2 chemokine, and the CXCR2 receptor that binds CXCL8. Interestingly, in several regions of the brain, low levels of CXCR2 are expressed on neurons, whereas high levels can be found in immune cells once they become activated under inflammatory conditions. Therefore, CXCR2 is yet another target of interest, which could act as a marker with specificity for neuroinflammation.

The list of potential targets can be made even longer and it is clear that a larger tool box of radioligands for a series of different targets is required to examine the postulated importance of inflammation in major CNS disorders.

\section{Compliance with ethical standards}

Conflict of interest Lars Farde is partly employed by AstraZeneca and owns shares in AstraZeneca.

Research involving human participants and/or animals This article does not contain any studies with human participants performed by the author.

\section{References}

1. Nimmerjahn A, Kirchhoff F, Helmchen F (2005) Resting microglial cells are highly dynamic surveillants of brain parenchyma in vivo. Science 308:1314-1318

2. Owen DR, Guo Q, Kalk NJ, Colasanti A, Kalogiannopoulou D, Dimber R et al (2014) Determination of [(11)C]PBR28 binding potential in vivo: a first human TSPO blocking study. J Cereb Blood Flow Metab 34:989-994. doi:10.1038/jcbfm.2014.46 